\title{
VELOCITY VECTOR FIELD VISUALIZATION OF FLOW IN LIQUID ACQUISITION DEVICE CHANNEL
}

\author{
John B. McQuillen ${ }^{1}$, David F. Chao ${ }^{1}$, Nancy R. Hall ${ }^{1}$, Nengli Zhang ${ }^{2, \dagger}$ \\ ${ }^{1}$ NASA Glenn Research Center, Cleveland, OH 44135, U.S.A. \\ ${ }^{2}$ Ohio Aerospace Institute at NASA Glenn Research Center, Cleveland, OH 44135, U.S.A. \\ \{john.b.mcquillen, david.f.chao, nancy.r.hall,nengli.zhang-1\}@nasa.gov
}

\begin{abstract}
Keywords: Velocity vector; Liquid acquisition device (LAD); flow pattern; CFD simulation; Vertically submerged screen channel; Gravity effects.

Abstract: $\quad$ A capillary flow liquid acquisition device (LAD) for cryogenic propellants has been developed and tested in NASA Glenn Research Center to meet the requirements of transferring cryogenic liquid propellants from storage tanks to an engine in reduced gravity environments. The prototypical mesh screen channel LAD was fabricated with a mesh screen, covering a rectangular flow channel with a cylindrical outlet tube, and was tested with liquid oxygen (LOX). In order to better understand the performance in various gravity environments and orientations at different liquid submersion depths of the screen channel LAD, a series of computational fluid dynamics (CFD) simulations of LOX flow through the LAD screen channel was undertaken. The resulting velocity vector field visualization for the flow in the channel has been used to reveal the gravity effects on the flow in the screen channel.
\end{abstract}

\section{INTRODUCTION}

As is well known for a space mission, liquid propellants have to be delivered from their storage tanks to an engine in a liquid state. It is easy to realize the delivery on earth using gravity to position liquid above the tank outlet that is located at the tank bottom. However, it is impossible to ensure the propellant drainage from the tank without vapor or pressurant gas entrainment in the low gravity environment of space, because of the uncertainty in the ullage position. One type of propellant management device, a liquid acquisition device (LAD), uses capillary flow and surface tension to acquire liquid has been developed and well characterized for storable (noncryogenic) propellants. Many of LADs have been built, tested and used for hypergolic propellants, such as nitrogen tetroxide $\left(\mathrm{N}_{2} \mathrm{O}_{4}\right)$ and monomethyl hydrazine $(\mathrm{MMH})$, but the development for use with cryogenic propellants has been lagging. LADs are custom designed to fulfill a unique set of requirements under the appropriate environmental mission conditions and demands; consequently, there is no universal design that satisfies all applications (Fester et al, 1976). Future space vehicles will require the use of non-toxic, cryogenic propellants, because of the performance advantages over the toxic hypergolic propellants and also because of the environmental and handling concerns (Kudlac and Jurns, 2006). Capillary-flow LADs have been well characterized for storable toxic propellants (Chato and M.T. Kudlac, 2002; Kudlac and Jurns, 2006), but there have been only a few LAD experiments with cryogenic propellants, including liquid oxygen (LOX). Recently, Zhang et al (2009) performed a CFD simulation for sub-cooled LOX flow in LAD channel test assembly in normal gravity environment. McQuillen et al (2012) conducted a series of CFD simulations of the LOX flows in LAD channel in different gravity environments for various flow rates. All of these simulations are for a "horizontally" submerged LAD channel in the LOX. This paper reports the CFD simulation results for "vertically" submerged in the LOX at normal gravity environment, revealing the gravity effects on the flow in the screen channel through velocity vector field visualizations produced by the simulation.

${ }^{\dagger}$ Corresponding author, Tel: 216-433-8750, Fax: 216-433-8050 


\section{LAD CHANNEL ASSEMBLY}

Under NASA's continuing Cryogenic Fluid Management (CFM) development program, a prototypical LAD screen channel assembly was designed and tested using LOX in NASA Glenn Research Center, as shown in Figure 1 left. The LOX flows into the channel assembly via the

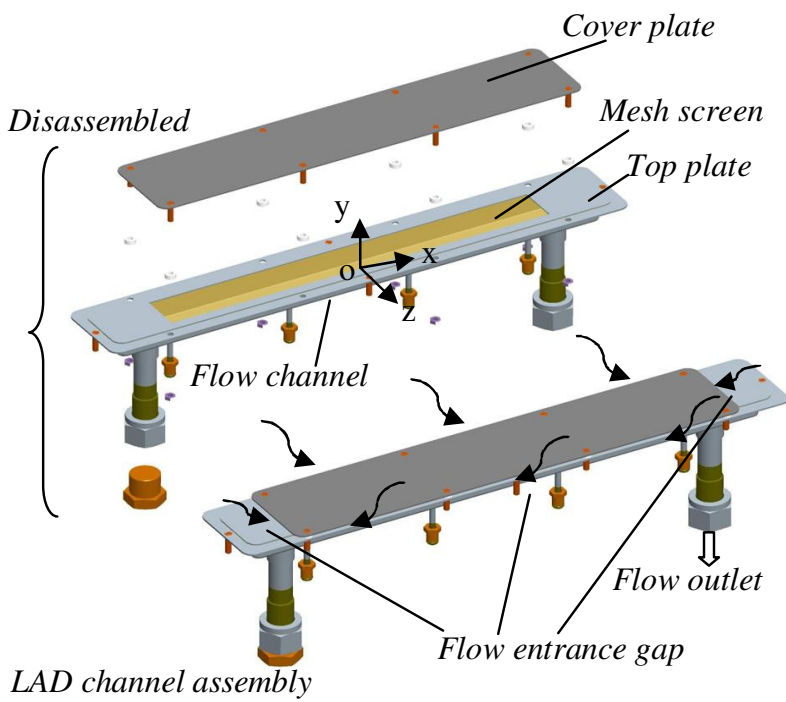

the gap between the cover plate and the mesh screen , so called entrance gap, from all directions, and then passes through the fine mesh screen to flow down of the channel assembly and then goes out. The mesh screen consists of stainless steel wires arranged in a 200x1400 Dutch Twill weave mesh. This geometry is shown in Figure 1 right.

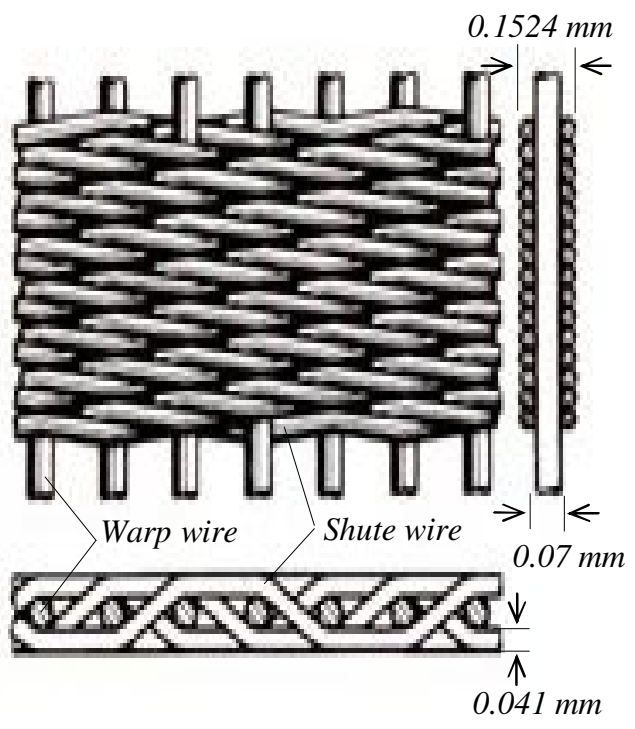

Figure 1: Prototypical LAD screen channel assembly and Geometry of Dutch Twill mesh.

The LOX flow path is shown schematically in Figure 2. Since most liquids, including LOX, perfectly wet stainless steel surfaces, the liquid wets the entire screen when the screen partially contacts the liquid pool, thus effectively blocking gas passage through the porous screen unless a critical pressure differential across the screen, is exceeded. This pressure differential is called the bubble point. The procedure for bubble-point test is

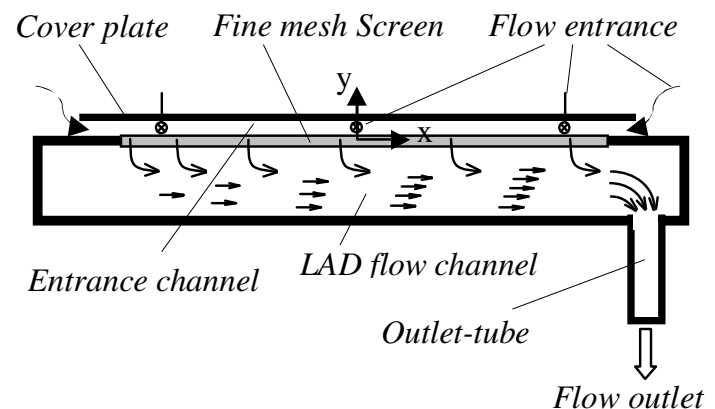

Figure 2: Fluid flow route in the LAD channel assembly. described in American Society for Testing and Materials Standard (ASTM) Method F316.

\section{CFD SIMULATION SETTINGS}

Since the Dutch Twill mesh screen is very thin compared to the LAD channel dimensions, the screen is approximated to a porous sheet and is handled as porous-jump in the CFD simulations of the LAD channel flow using FLUENT. Two primary parameters, the face permeability, $\alpha$, and the pressure jump coefficient, $C_{2}$, define the porousjump in FLUENT, which can be determined experimentally. The relevant reviews and calculation methods have been given by Zhang et al. (2009) and McQuillen et al. (2012).

In order to simulate the cases of vertically partial submersions of the LAD channel in LOX, several geometry models have been established, including full submersion and partial submersion depths of the mesh screen, such as $1 / 3,1 / 2$, and $2 / 3$ of the screen length submerged. For the partially submerged cases, the exposed portion of the mesh 
screen was taken as a solid wall, as shown in Figure 3. This is reasonable because no liquid or vapor can flow through this portion of the screen provided that the local pressure drop between the vapor and the LOX does not exceed the bubble point.

The pressure-based solver was used, with a steady implicit formulation. The three-dimensional simulations used the Green-Gauss cell based gradient and the standard k-epsilon model for the turbulent flow with the following constants: The coefficient of first order turbulent dissipation rate in the dissipation rate equation, $C_{l \varepsilon}=1.44$; the coefficient of second order turbulent dissipation rate in the dissipation rate equation $C_{2 \varepsilon}=1.92$; the turbulent viscosity coefficient $C_{\mu}=0.09$; the turbulent Prandtl number for turbulent kinetic energy, $\sigma_{k}=1.0$; and the turbulent Prandtl number for dissipation rate of turbulent kinetic energy, $\sigma_{\varepsilon}=1.3$.

The operating conditions were set to a system pressure $=1620269 \mathrm{~Pa}, \mathrm{~T}=89 \mathrm{~K}$, and gravitational acceleration vectors were set at $g_{x}=g_{z}=0.0 \mathrm{~m} / \mathrm{s}^{2}$, and $g_{y}=-9.81 \mathrm{~m} / \mathrm{s}^{2}$ for the horizontally-oriented completely submersion cases, while $g_{y}=g_{z}=0.0$ $\mathrm{m} / \mathrm{s}^{2}$, and $g_{x}=-9.81 \mathrm{~m} / \mathrm{s}^{2}$ for vertically-oriented completely and partially submerged cases.

The 'pressure-outlet' type was selected as the boundary condition at the flow outlet, and set to a gauge pressure of $0 \mathrm{~Pa}$ with a backflow modified turbulent viscosity of $0.001 \mathrm{~m}^{2} / \mathrm{s}$. The 'pressure-inlet' type boundary condition was selected at the flow inlet surface with corresponding values of total gauge pressure and modified turbulent viscosity of $0.001 \mathrm{~m}^{2} / \mathrm{s}$ to obtain a mass flow rates within the range of $0.0455 \mathrm{~kg} / \mathrm{s}(0.1 \mathrm{lbs} / \mathrm{s}) \sim 0.1818 \mathrm{~kg} / \mathrm{s}(0.4$ $\mathrm{lbs} / \mathrm{s})$. It should be noted that all the gauge pressures are relative to the operating pressure.
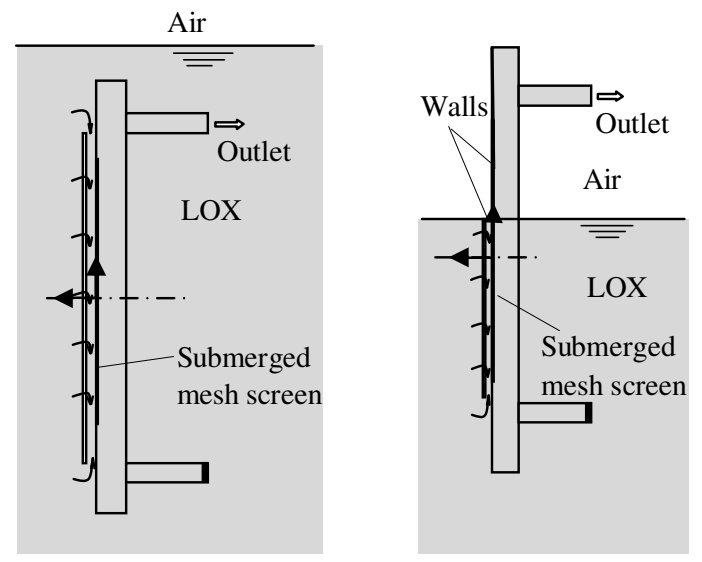

Figure 3: Vertically submerged LAD channel assembly

\section{SIMULATION RESULTS}

Twenty four cases of vertically-oriented submerged LAD channel, including six complete submerged, seven $2 / 3$ submerged screen length, five $1 / 2$ submerged screen length, and six $1 / 3$ submerged screen length cases, were simulated. Two cases of horizontally-oriented submerged LAD channel were simulated for comparison with the corresponding vertically-oriented completely submerged cases. The simulation results show that for the completely submerged cases, the mass flow rate passing through the LAD channel is not affected by the orientation of the channel. In fact, the total static pressure drop between the inlets and outlet, $\Delta p_{i-o}$, and submerged portion of the screen determines the flow rate. For example, at the cases of completely submerged channel, the value of $\Delta p_{i-o}$ is proportional to the mass flow rate and independent of channel orientation However, as more of the screen becomes exposed in the vertically-oriented submerged cases, a larger $\Delta p_{i-o}$ is needed to achieve the same mass flow rate. As is well known, the flow pattern in the channel directly reflects the effects of gravity on the channel performance. In order to ascertain the effects of the channel orientation or gravity on the LAD channel performance, the velocity vector fields are visualized and compared to reveal the flow pattern characteristics.

\subsection{Velocity vector field visualization}

For the case of vertically-oriented, completely submerged channel, the hydrostatic pressures in both entrance gap and LAD flow channel are the same; therefore, gravity has no effect on the flow in the channel. The flow pattern in the channel is no different between the completely submerged cases horizontally and vertically oriented channels. For example, the velocity vectors near the outlet tube for both cases are almost identical, as shown in Figure 4. The velocity vectors for completely submerged channels along the channel length at the sections of axial distance of $x=-0.16 \mathrm{~m}, x=0 \mathrm{~m}$, and $x=0.16$ $\mathrm{m}$, are also identical for horizontally and vertically oriented channels, respectively, as shown in Figures $5 \mathrm{a}, 45 \mathrm{~b}$, and $5 \mathrm{c}$, in which the left ones are for horizontal orientation while the right ones for vertical orientation. However, gravity affects the flow pattern in the flow channel when a portion of the screen is exposed. At the same $\Delta p_{i-o}$, the amount of exposed screen length is proportional to the hydrostatic pressure drop between the entrance gap 

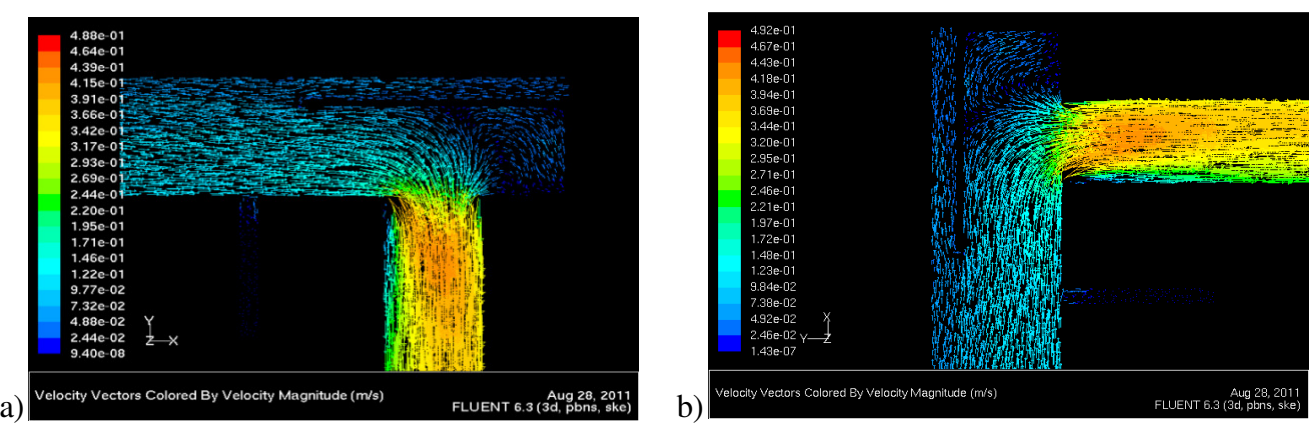

Figure 4: Velocity vectors in the completely submerged channel near the outlet tube: a) horizontally oriented; b) vertically oriented channel.

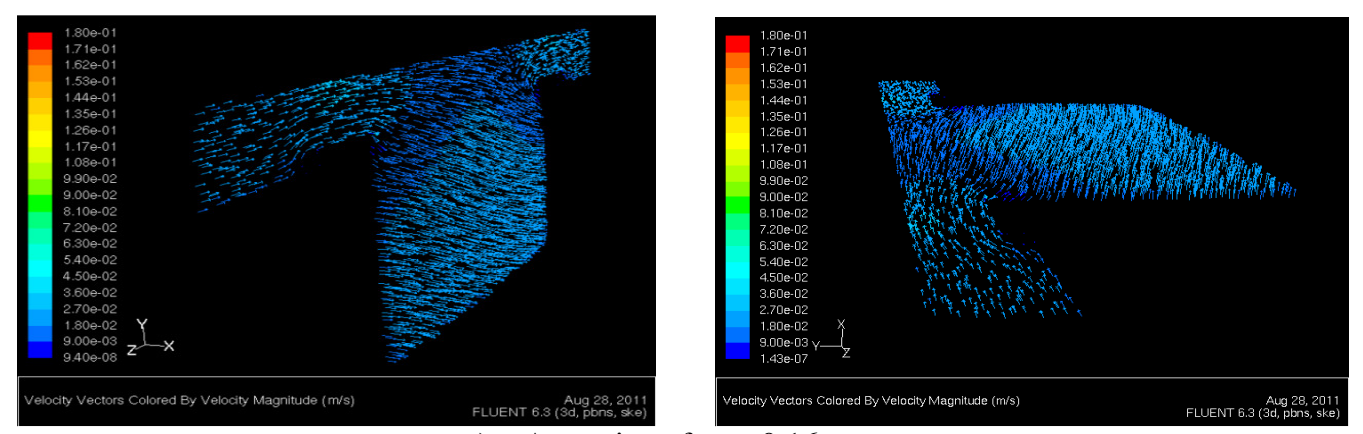

a) At section of $x=-0.16 \mathrm{~m}$.
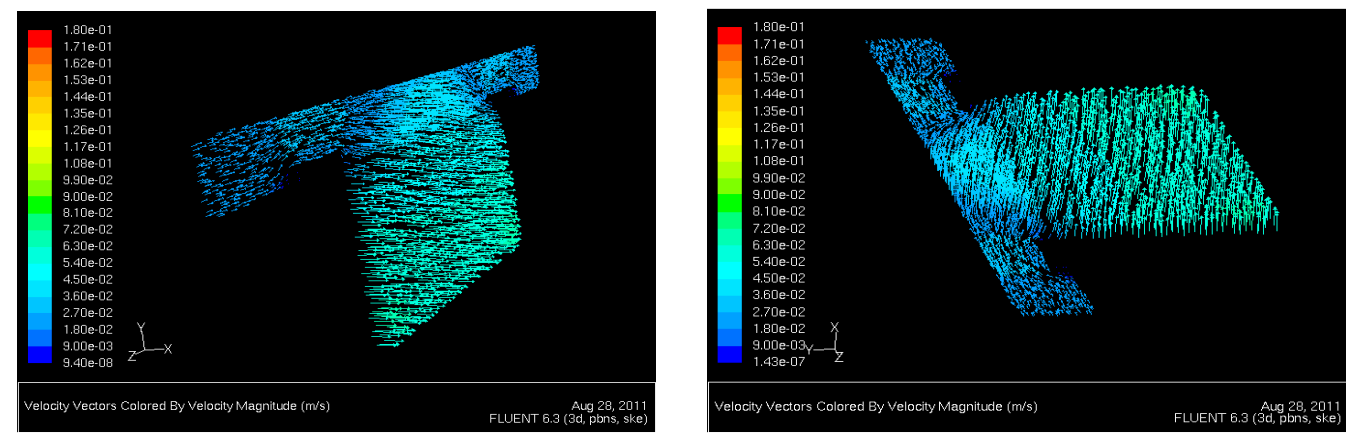

b) At section of $x=0.0 \mathrm{~m}$.
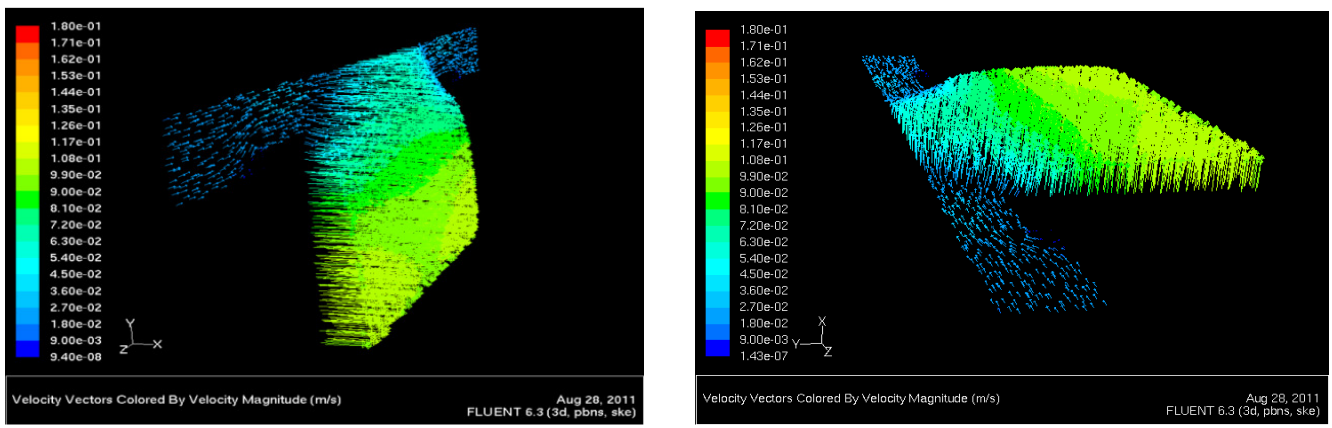

c) At section of $\mathrm{x}=0.16 \mathrm{~m}$.

Figure 5: Velocity vectors at different sections. 
and the LAD flow channel; consequently, when the screen exposed portion increases, the resistance of the flow passing though the flow channel becomes larger. As a result, the flow in the flow channel by the outlet tube is different for the cases of various screen exposures. For the case of vertically-oriented, completely submerged channel, the flow has higher velocity in the channel and a vortex occurs at the flow channel end by the outlet tube, as shown in Figure 6a, while for the cases of vertically-oriented exposed screen half length, the flow enters into the outlet tube without generating a vortex, because the higher hydrostatic pressure drop reduces the flow velocity in the flow channel, as shown in Figure $6 \mathrm{~b}$. It should also be noted that for cases of completely submerged channel and half submerged screen length, the flow direction passing though the screen is different at the bottom end of the flow channel, as shown in Figure 7.

\subsection{Velocity and Mass flow rate change}

The CFD simulation results show that the flow velocity and mass flow rate in the channel are continuously augmented along the submerged channel length (x-axis direction) when the LOX passes through the screen and then flows toward the outlet tube in the flow channel. The typical velocity vectors distributions at different sections for the cases of completely submerged and half submerged screen length are shown in Figure 8. It is noted that when the LOX flows in the exposed screen portion of the channel, the flow rate maintains constant because no LOX is added into the channel through the screen anymore.

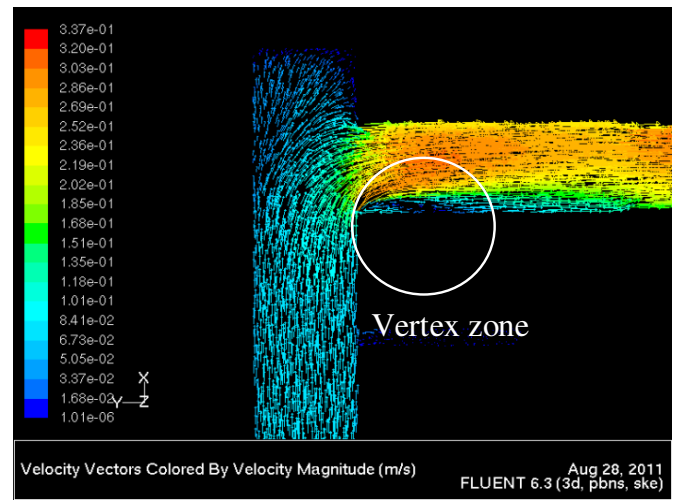

a) In case of vertically full submersion

\section{CONCLUSIONS}

In virtue of the velocity vector field visualization, the flow pattern characteristics in the capillary flow LAD channel are revealed and the performance of the LAD channel at different submersion cases in normal gravity can be evaluated.

1. The flow patterns in the completely submerged LAD channel are identical between the cases of horizontal and vertically-oriented channels.

2. The gravity effects flow pattern in the flow channel when as of the amount of exposed screen length is varied. At the bottom end of the channel, far away from the outlet tube, the hydrostatic pressure drop reverses the flow direction of the LOX passing the screen, while at the top end of the channel, the flow smoothly enters into the outlet tube without vortex, which is contrary to one in the completely submerged channel.

3 . The flow velocity and mass flow rate in the channel are continuously augmented along the channel length (x-axis direction) when the LOX passes through the screen and then flows toward the outlet tube in the flow channel. However, when the LOX flows in the exposed screen portion of the channel, the flow rate is constant.

\section{REFERENCES}

Chato, D. J. and Kudlac, M. T., 2002. In: $38^{\text {th }}$ AIAA/ASME/SAE/ASEE Joint Propulsion Conference \& Exhibit, Indianapolis, IN, see also Paper AIAA 2002-3983.

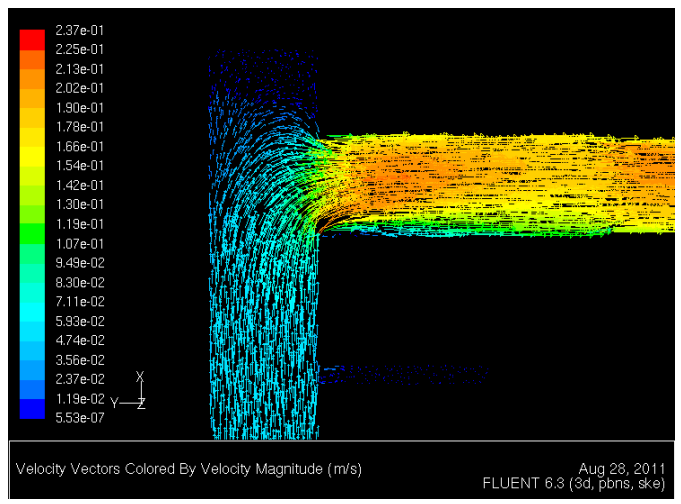

b) In case of vertically half screen submersion

Figure 6: Velocity vectors in the channel near the outlet tube for cases of vertically submerged. 


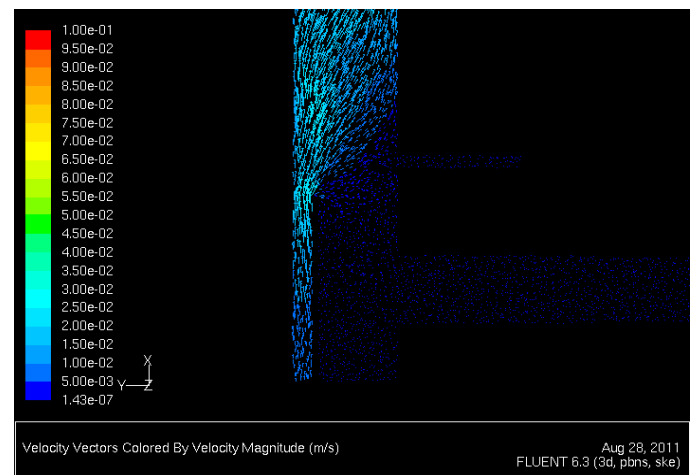

In case of vertically full submersion

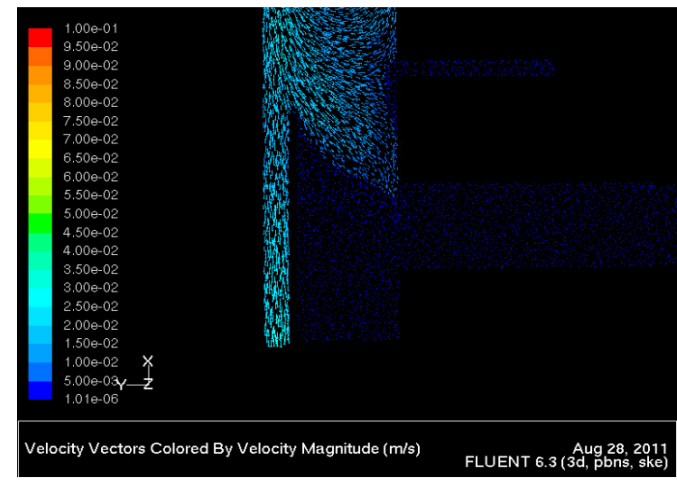

In case of vertically half screen submersion

Figure 7: Velocity vectors in the channel at bottom end for the cases of vertically submerged.

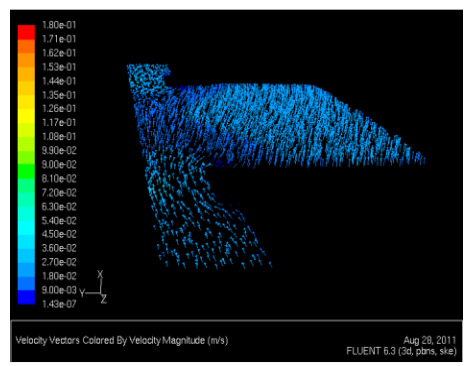

At section of $\mathrm{x}=-0.16 \mathrm{~m}$

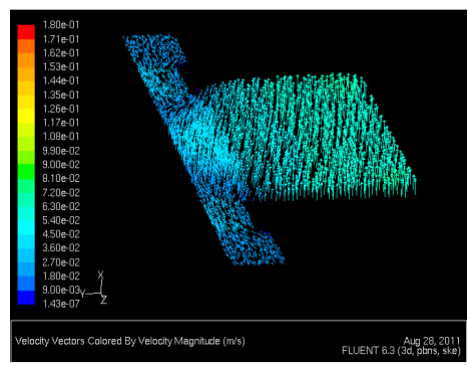

At section of $\mathrm{x}=0 \mathrm{~m}$

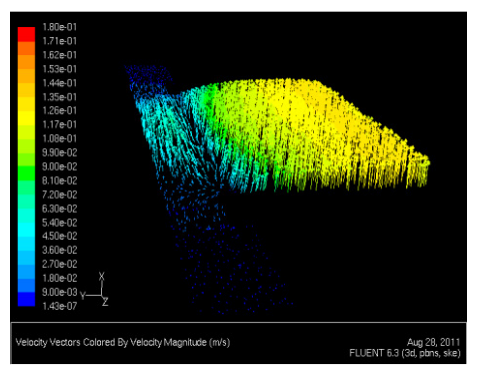

At section of $\mathrm{x}=0.24 \mathrm{~m}$

a) In case of vertically full submersion

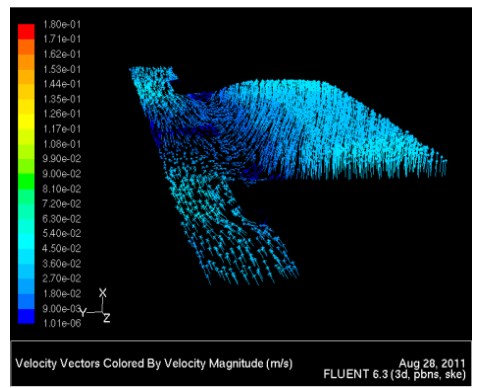

At section of $\mathrm{x}=-0.16 \mathrm{~m}$

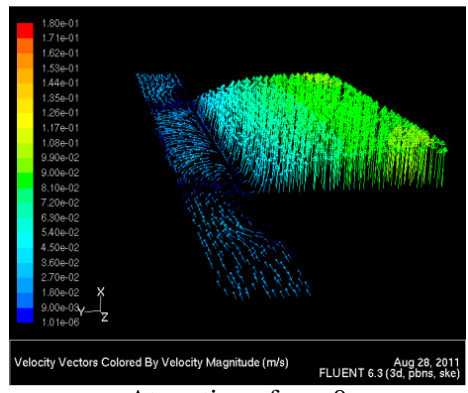

At section of $\mathrm{x}=0 \mathrm{~m}$

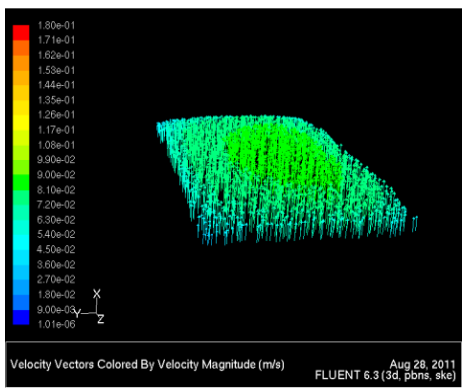

At section of $\mathrm{x}=0.24 \mathrm{~m}$

b) In case of vertically half screen submersion.

Figure 8: Velocity vectors distributions in the channel assembly at $\Delta \mathrm{p}_{\mathrm{i}-\mathrm{o}}=400 \mathrm{~Pa}$.

Fester, D. A., Villars, A. J. and Uney, P.E., 1976. Journal of Spacecraft and Rocket ,13: 522527.

Kudlac, M. T. and Jurns, J. M., 2006. In: $42^{\text {nd }}$ AIAA/ASME/SAE/ASEE Joint Propulsion Conference \& Exhibit, Sacramento, CA, see also Paper AIAA 2006-5054.
McQuillen, J. B., Chato, D. J., Motil, B. J., Doherty, M. P., Chao, D. F. and Zhang, N., 2012. Journal of Porous Media (in print).

Zhang, N., Chato, D. J., . McQuillen, J. B., Motil, B. J. and Chao, D. F., 2009. World Academy of Science, Engineering and Technology, 58: 1180-1185. 